Abgesehen von den beiden besprochenen Fällen aus den Jahren 1861 und 1898 hat $R$ Coronae, soweit die Beobachtungen reichen, niemals seine normale Helligkeit uberschritten.

3) In den verschiedenen Minima 1) ist die kleinste Helligkeit von $R$ Coronae ganz außerordentlich verschieden, und es lassen sich keinerlei Gesetzmäßigkeiten feststellen. Es gibt Minima, in denen die Helligkeit gegenuber der normalen nur um ein bis zwei Größenklassen abnimmt; solche sind namentlich in den letzten Jahrzehnten häufiger aufgetreten, nämlich $188_{5}$ (kleinste Größe $m=7.7$ ), I 900 $(m=7.7)$, $1903(m=8.3), 1904(m=7.2)$. In andern Minima wird der Veränderliche außerordentlich schwach. Die schwächste beobachtete Größe ist 15.0 (im Jahre 1872 von Schönfeld geschätzt), doch ist es nicht ausgeschlossen, daß der Veränderliche zuweilen noch schwächer wird. Die sehr schwachen Minima sind zumeist nur sehr unvollständig beobachtet.

Auch die Dauer der Minima ist außerordentlich verschieden. Die kürzesten sind das erste von $1895(m=9.5)$ und das von rgo4 ( $m=7.2$ ), die beide nur etwa je $z w e i$ Monate in Anspruch nahmen. Das Minimum von längster Dauer währte von Ende 1863 bis in die zweite Hälfte des Jahres I874, d. h. während dieser ganzen Zeit hat der Veränderliche niemals die normale Größe erreicht; allerdings stieg die Helligkeit in dem genannten Zeitintervall mehrmals in sekundären Maxima bis zur siebenten oder achten Größe, einmal sogar bis zur Größe 6.6 empor.

Ebenso verschieden wie die Dauer der Minima ist auch die Form der Lichtkurve während des Verlaufes derselben. Charakteristisch für den Beginn der Minima ist zumeist ein außerordentlich rasches, gleichmäßiges Abnehmen der Helligkeit; als Beispiele führe ich folgende Zahlen an:

I 859 Abnahme der Helligkeit um $4 \frac{1}{2}$ Größenkl. in $20^{d}$

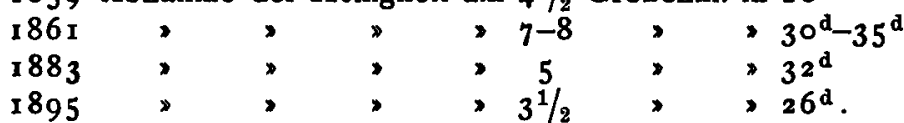

Sehr selten ist beim Beginn eines Minimums ein langsames Sinken der Helligkeit; ein solches kommt in der Regel nur bei unbedeutenden Minima vor, so bei denen von 1885 und I903. Verháltnismäßig langsam war der Abfall der Lichtkurve auch bei dem recht bedeutenden Minimum von I887 ( $m=$ I I.2).

Das Wiederanwachsen der Helligkeit zur normalen Größe geht meist viel langsamer vor sich als die Abnahme.
Der aufsteigende Teil der Lichtkurve ist häufig unregelmäßig und zeigt sekundäre Maxima und Minima. Zuweilen geschieht aber die Wiederzunahme der Helligkeit ebenso plötzlich wie die Abnahme, so bei dem tiefen Minimum von 1861 $(m=13.2)$. In solchen Fällen hat die Lichtkurve, abgesehen von den zeitlichen Verhältnissen, eine gewisse Ähnlichkeit mit der von Algolsternen.

Zwischen dem Sinken unter die normale Helligkeit und dem Wiederanwachsen zu derselben vollführt der Veränderliche häufig die merkwürdigsten und unregelmäßigsten Lichtschwankungen, mit zahlreichen sekundären Maxima und Minima.

Irgendwelche Gesetzmäßigkeiten in der Zeit des Eintretens der Minima habe ich nicht auffinden können. Wo sich $a b$ und zu solche zu zeigen scheinen, werden sie stets durch den weiteren Verlauf der Lichtschwankungen wieder umgestoßen.

Die vorstehenden Betrachtungen lassen sich kurz in folgende Sätze zusammenfassen:

I) $\mathbf{R}$ Coronae ist oft lange Zeit hindurch von konstanter Helligkeit, und zwar besitzt er dann stets sehr nahe die Größe 6.0. Kleine, insgesamt 0.2 bis 0.3 Größenklassen betragende Änderungen dieser normalen Größe sind angedeutet.

2) Ein Anwachsen der Helligkeit von $\mathbf{R}$ Coronae uber die normale Größe findet jedenfalls nur ăußerst selten statt. Kein einziger derartiger Fall ist durch gleichzeitige Schätzungen verschiedener Beobachter oder auch nur durch eine größere Beobachtungsreihe eines Beobachters sicher verbürgt.

3) Die Zeiten konstanter Helligkeit werden anscheinend regellos unterbrochen durch zahlreiche Minima von verschiedenster Dauer und verschiedenster Minimalhelligkeit. Die Helligkeitsabnahme bei Beginn eines Minimums geschieht zumeist sehr schnell, die Wiederzunahme am Ende eines Minimums meist weit langsamer. Dazwischen liegen Lichtschwankungen von größter Unregelmäßigkeit.

Von den bisher bekannten Veränderlichen scheint nur RY Sagittarii eine ähnliche Lichtkurve wie $\mathbf{R}$ Coronae zu besitzen. Eine gewisse Verwandtschaft besteht vielleicht auch zwischen $\mathbf{R}$ Coronae und $\mathbf{X}$ Persei.

Ehe man daran geht, Hypothesen zur Erklärung der von $\mathbf{R}$ Coronae dargebotenen merkwürdigen Erscheinungen aufzustellen, ist eine eingehende Untersuchung des Spektrums dieses Sterns dringend nötig. Was bisher über dasselbe, sowie tuber die Radialgeschwindigkeit bekannt geworden ist, habe ich in A. N. 4129 zusammengestellt.

Potsdam, Astrophysikalisches Observatorium, I908 April I 4.

\section{H. Ludendorff.}

1) Unter einem Minimum ist hier stets ein solcher Teil der Lichtkurve verstanden, der sich vom Beginne des Sinkens unter die normale Helligkeit bis zur Wiedererreichung derselben erstreckt.

\section{Uber die Parallaxen der Nebel GC 4964 und NGC. 7027. Von Einar Huss.}

In den Astr. Nachr. $326 \mathrm{I}$ und 3190 hat Herr Prof. letzteren Falle die Resultate der Rechnung mitgeteilt, nämlich Wilsing in Potsdam eine Reihe Beobachtungen veröffentlicht, die er unternommen hat, um die Parallaxe der beiden Nebel

GC. 4964 und NGC. 7027 zu bestimmen. Er hat nur im in bezug auf den einen und

$$
\pi=-0.083 \pm 0.025
$$




$$
\pi=-0.172 \pm 0.068
$$

auf den anderen Vergleichstern. Dieses Resultat erscheint zunächst sehr befremdend, weil die beiden Vergleichsterne I I. Größe sind. Prof. Wilsing hatte bei der Berechnung den Einfluß einer etwaigen Differenz der Aberrationskonstante für das Hauptobjekt und für die Vergleichsterne nicht mitgenommen, wonach es möglich wăre, den großen negativen Wert der Parallaxe bei Einfuhrung dieser Korrektion ein wenig zu reduzieren.

Die Eigenbewegung der beiden Objekte ist verschwindend klein, was auch aus dem Resultat von Prof. Wilsing hervorgeht, indem er fur den Nebel NGC. 7027 in bezug auf die beiden Vergleichsterne die Werte

$$
y=+0.075 \pm 0.063 \text { und } y_{1}=-0.070 \pm 0.063
$$

gefunden hat. Im folgenden ist deshalb die Eigenbewegung vernachlässigt worden.

\section{Der Nobel GC. 4964.}

Wir führen folgende Bezeichnungen ein :

$\sigma=$ Korrektion der Distanz zwischen dem Objekte und den Vergleichsternen,

$\pi=$ Parallaxe,

$x=$ Korrektion der Aberrationskonstante und

$n=$ Differenz zwischen der beobachteten Distanz und dem Mittelwerte der Distanzen.

Wir erhalten dann aus den Beobachtungen von Prof. Wilsing 3 I Bedingungsgleichungen futr jeden Vergleichstern. Sodann werden die Normalgleichungen:

$$
\begin{gathered}
\text { I. Nebel-Stern } 3 . \\
+31.000 \sigma_{1}-11.289 \pi-16.642 x=-2.100 \\
+17.072+2.470=+0.095 \\
\text { II. Nebel-Stern } 6 . \\
+31.000 \sigma_{2}-16.586 \pi-1.805 x=-0.250 \\
+14.160-2.762=-0.866 \\
+8.872=+1.282 .
\end{gathered}
$$

Daraus sind folgende Resultate zu erhalten:

$$
\text { I. }
$$

$$
\pi=-0.112 \pm 0.098
$$$$
x=+0.109 \pm 0.080
$$

bezw.

II.

$$
\begin{aligned}
& x=-0.077 \pm 0.066 \\
& x=-0.093 \pm 0.140 .
\end{aligned}
$$

Diese Resultate, insbesondere der Wert der Aberration, sind ungünstig, ja, einander fast widersprechend. Die Erklärung dieses Sachverhalts ist darin zu suchen, daß die Verbindungslinien des Objekts mit den Sternen beinahe senkrecht zueinander stehen. Die Berechnung wird darum im ersten Falle einen schlechten Wert für $\pi$ und in dem anderen Falle für $x$ geben. Betrachten wir aber die beiden Gruppen von Bedingungsgleichungen als eine einzige Gruppe von 62 Gleichungen, vier Variable enthaltend, also von der Form:

$$
\sigma_{1}+\sigma_{2}+a_{1} \pi+a_{2} x=n
$$

so werden wir bessere Werte bekommen. Die Normalgleichungen sind in diesem Falle:

$$
\begin{array}{r}
+31.000 \sigma_{1}+0.000 \sigma_{2}-11.289 \pi-16.642 x=-2.100 \\
+31.000-16.586-1.805=-0.250 \\
+31.232-0.292=-0.771 \\
+20.712=+2.418
\end{array}
$$

woraus :

$$
\pi=-0.063 \pm 0.05^{\circ} \quad x=+0.069 \pm 0.063
$$

die wir als definitive Werte annehmen dürfen.

Der Webbsche Nobel NGC. 7027.

Auch hier sind die Vergleichsterne so gewählt, daß ihre Verbindungslinien mit dem Objekt senkrecht zueinander stehen. Indem wir wiederum die zwei Gruppen von Bedingungsgleichungen zusammennehmen, erhalten wir folgende Normalgleichungen:

$$
\begin{array}{r}
+34.000 \sigma_{1}+0.000 \sigma_{2}-9.852 \pi-16.919 x=+1.050 \\
+32.000-15.207+4.555=+2.270 \\
+31.133-0.696=-3.946 \\
+24.635=+0.485
\end{array}
$$

und daraus als definitive Werte:

$$
\begin{aligned}
& \pi=-0.119 \pm 0.021 \\
& x=+0.018 \pm 0.025
\end{aligned}
$$

welche von Prof. Wilsings Resultat nur wenig abweichen.

Es ist uns also auf diesem Wege zwar nicht gelungen den großen negativen Wert, den Prof. Wilsing für die
Parallaxe des Nebels NGC. 7027 gefunden hat, bedeutend zu reduzieren, aber wir haben hiermit die relative Parallaxe der beiden Nebel konstatiert. Wenn wir nämlich bedenken, daß die beiden Objekte fast in derselben Gegend des Himmels liegen und von Prof. Wilsing beinahe gleichzeitig beobachtet worden sind, können wir es als festgestellt ansehen, daß der Webbsche Nebel NGC. 7027 entfernter ist, als der Nebel GC. 4964 .

Stockholm, rgo8 April 4. 